TRANSACTIONS OF THE

AMERICAN MATHEMATICAL SOCIETY

Volume 350, Number 8, August 1998, Pages 3119-3130

S 0002-9947(98)02294-6

\title{
COMPLICATED DYNAMICS OF PARABOLIC EQUATIONS WITH SIMPLE GRADIENT DEPENDENCE
}

\author{
MARTINO PRIZZI AND KRZYSZTOF P. RYBAKOWSKI
}

ABstract. Let $\Omega \subset \mathbb{R}^{2}$ be a smooth bounded domain. Given positive integers $n, k$ and $q_{l} \leq l, l=1, \ldots, k$, consider the semilinear parabolic equation

$\begin{aligned} \text { (E) } u_{t} & =u_{x x}+u_{y y}+a(x, y) u+\sum_{l=1}^{k} a_{l}(x, y) u^{l-q_{l}}\left(u_{y}\right)^{q_{l}}, & & t>0,(x, y) \in \Omega, \\ u & =0, & & t>0,(x, y) \in \partial \Omega\end{aligned}$

where $a(x, y)$ and $a_{l}(x, y)$ are smooth functions. By refining and extending previous results of Poláčik we show that arbitrary $k$-jets of vector fields in $\mathbb{R}^{n}$ can be realized in equations of the form $(\mathrm{E})$. In particular, taking $q_{l} \equiv 1$ we see that very complicated (chaotic) behavior is possible for reaction-diffusionconvection equations with linear dependence on $\nabla u$.

\section{INTRODUCTION}

Let $\Omega \subset \mathbb{R}^{2}$ be a smooth bounded domain. We consider the semilinear parabolic equation

$$
\begin{aligned}
u_{t} & =u_{x x}+u_{y y}+a(x, y) u+f\left(x, y, u, u_{y}\right), & & t>0,(x, y) \in \Omega, \\
u & =0, & & t>0,(x, y) \in \partial \Omega,
\end{aligned}
$$

where $a: \mathbb{R}^{2} \rightarrow \mathbb{R}$ is continuous and $f: \mathbb{R}^{4} \rightarrow \mathbb{R},(x, y, s, w) \mapsto f(x, y, s, w)$, is a smooth function (more precisely, $f$ is continuous together with all its partial derivatives with respect to $(s, w))$.

Set $X=L^{p}(\Omega), p>2$, and let $A: W^{2, p}(\Omega) \cap W_{0}^{1, p}(\Omega) \rightarrow X$ be the linear operator defined as

$$
-A u=u_{x x}+u_{y y}+a(x, y) u .
$$

Being a sectorial operator, $A$ generates the family $X^{\alpha}, \alpha \geq 0$, of fractional power spaces. Choosing $\alpha$ with $\frac{1}{2}+\frac{1}{p}<\alpha<1$, we have that $X^{\alpha} \subset C^{1}(\bar{\Omega})$ with continuous inclusion.

Define the Nemitski operator $\hat{f}: X^{\alpha} \rightarrow X$ by

$$
\hat{f}(u)(x, y)=f\left(x, y, u(x, y), u_{y}(x, y)\right) .
$$

Received by the editors May 16, 1996.

1991 Mathematics Subject Classification. Primary 35K20; Secondary 35B40.

Key words and phrases. Center manifolds, jet realization, parabolic equations, chaos.

The research of the second author was supported, in part by MURST $40 \%$ and in part by the project Reaction-Diffusion Equations, Contract no. ERB CHRX CT 930 409, of the Human Capital and Mobility Programme of the European Communities.

(C)1998 American Mathematical Society 
Then equation (1) can be rewritten in the form

$$
u_{t}+A u=\hat{f}(u) .
$$

It is well known that (2) defines a dynamical system (more precisely, a local semiflow) on $X^{\alpha}$.

In his recent paper [11], Poláčik showed that the dynamics of equation (2) can be very complicated. He proved in fact that a dense set of dynamics of ordinary differential equations on $\mathbb{R}^{n}$ can be 'simulated' by such parabolic equations.

In order to describe Poláčik's results, let us introduce some terminology. Let $X_{1}:=\operatorname{ker} A$, and suppose $X_{1} \neq\{0\}$. Since $A$ has compact resolvent, $X_{1}$ is finite dimensional, and since $A$ as an operator in $L^{2}(\Omega)$ is selfadjoint, we can find an $L^{2}(\Omega)$-orthonormal basis of eigenfunctions $\phi_{1}, \ldots, \phi_{n}$ for $X_{1}$ so that the spectral projection $P$ for the spectral set $\{0\}$ is the orthogonal projection given by

$$
P u=\sum_{i=1}^{n} \phi_{i} \int_{\Omega} u \phi_{i} d x .
$$

The space $X$ is then decomposed as the sum of two $A$-invariant subspaces, namely $X=X_{1} \oplus X_{2}$, where $X_{2}=\operatorname{ker} P$.

Let $J_{0}^{k}\left(X_{1}\right)$ denote the set of all $k$-jets on $X_{1}$ mapping 0 to itself. Equivalently, $h \in J_{0}^{k}\left(X_{1}\right)$ if and only if $h$ is a polynomial on $X_{1}$ of order $\leq k$ with $h(0)=0$. Using the basis $\phi_{1}, \ldots, \phi_{n}$ of $X_{1}$, we see that $h$ can also be regarded as a polynomial on $\mathbb{R}^{n}$ of order $\leq k$.

Now Poláčik proved (see [11, Theorem 2.2] that, given any positive integers $n$ and $k$, there is a convex smooth bounded domain $\Omega \subset \mathbb{R}^{2}$ and a smooth function $a=a(x, y)$ such that the corresponding operator $A$ has $n$-dimensional kernel $X_{1}$. Moreover, there is a neighborhood $\mathcal{B}$ of zero in $J_{0}^{k}\left(X_{1}\right)$ such that every jet $h \in \mathcal{B}$ can be realized on a local center manifold of equation (2) for an appropriate choice of the nonlinearity. More precisely, if $h \in \mathcal{B}$ then there is a nonlinearity $f=f(x, y, w)$ of the form

$$
f(x, y, w)=\sum_{l=1}^{k} a_{l}(x, y) w^{l}, \quad \text { with } a_{l} \in H^{2}(\Omega),
$$

there is an open neighborhood $U$ of zero in $X_{1}$ and a $C^{k}$-map $\sigma: U \rightarrow X_{2} \cap X^{\alpha}$ with $\sigma(0)=0$ with the properties that the manifold

$$
W=\left\{u_{1}+\sigma\left(u_{1}\right) \mid u_{1} \in U\right\}
$$

is locally invariant with respect to (2) and the $k$-th order Taylor polynomial at zero of the map

$$
u_{1} \mapsto P \hat{f}\left(u_{1}+\sigma\left(u_{1}\right)\right): U \rightarrow X_{1}
$$

is equal to $h$. The proof of [11, Theorem 2.2] actually implies that a dense set of vector fields of ordinary differential equations on a bounded set of $\mathbb{R}^{n}$ can be realized (up to flow equivalence) on local center manifolds of equations of the form (2) with $f$ of the form (3). See [11, Corollary 2.5] for a precise statement.

The form (3) of the nonlinearity $f$ means that equation (1) (or, equivalently, equation (2)) depends on high powers of the gradient of the solution $u$. On the other hand, when modelling scientific phenomena by equation (1), one usually tries to make the convection terms (i.e. terms depending on $\nabla u$ ) as simple as possible, e.g. linear in $\nabla u$. Therefore the question arises if such systems can also exhibit 
complex dynamics. The purpose of this paper is to give a positive answer to this question. More precisely, by refining some crucial arguments in [11] we shall prove that the jet realization result [11, Theorem 2.2] holds for nonlinearities of the form

$$
f(x, y, s, w)=\sum_{l=1}^{k} a_{l}(x, y) s^{p_{l}} w^{q_{l}}, \quad \text { with } a_{l} \in H^{2}(\Omega),
$$

where for every $l$ with $1 \leq l \leq k, q_{l}$ is an arbitrarily prescribed integer with $1 \leq q_{l} \leq l$ and $p_{l}:=l-q_{l}$. In particular, choosing $q_{l} \equiv 1$ we obtain a jet realization result for nonlinearities with linear gradient dependence. This gives a positive answer to a question posed by Poláčik in [11]. We also prove that the above mentioned vector field realization result [11, Corollary 2.5] continues to hold for nonlinearities with prescribed (e.g. linear) gradient dependence.

\section{THE MAIN RESULTS}

The aim of this section is the proof of the following result:

Theorem 1. For $n$ and $k \in \mathbb{N}$, and arbitrary integers $q_{1}, \ldots, q_{k}$ such that $1 \leq$ $q_{l} \leq l$ for $l=1, \ldots, k$, let $\mathcal{E}=\mathcal{E}\left(q_{1}, \ldots, q_{k}\right)$ be the set of all functions $f: \mathbb{R}^{4} \rightarrow \mathbb{R}$ of the form

$$
f(x, y, s, w)=\sum_{l=1}^{k} a_{l}(x, y) s^{l-q_{l}} w^{q_{l}}, \quad(x, y, s, w) \in \mathbb{R}^{4},
$$

where $a_{l} \in H^{2}(\Omega)$ for $l=1, \ldots, k$.

There exist a convex bounded domain $\Omega \subset \mathbb{R}^{2}$ with smooth boundary and a $C^{\infty}$ function $a(x, y)$ such that:

1. The operator $A$ has n-dimensional kernel;

2. There is an open neighborhood $\mathcal{B}$ of 0 in $J_{0}^{k}\left(X_{1}\right)$ such that every jet $h \in \mathcal{B}$ can be realized in (1) by a nonlinearity $f \in \mathcal{E}$; that is, every $h \in \mathcal{B}$ can be realized in an equation of the form

$$
\begin{aligned}
u_{t} & =u_{x x}+u_{y y}+a(x, y) u+\sum_{l=1}^{k} a_{l}(x, y) u^{l-q_{l}}\left(u_{y}\right)^{q_{l}}, & & t>0,(x, y) \in \Omega, \\
u & =0, & & t>0,(x, y) \in \partial \Omega .
\end{aligned}
$$

Remark. Choosing $q_{l}=l$ for all $l=1, \ldots, k$, we obtain Poláčik's result [11, Theorem 2.2]. On the other hand, choosing $q_{l}=1$ for all $l=1, \ldots, k$, we obtain a jet realization result for nonlinearities which are polynomials in $u$ and which are linear functions of $u_{y}$.

Theorem 1 can be strengthened as follows:

Theorem 2. For $n$ and $k \in \mathbb{N}$, and arbitrary integers $q_{1}, \ldots, q_{k}$ such that $1 \leq$ $q_{k} \leq l$ for $l=1, \ldots, k$, there exist a convex bounded domain $\Omega \subset \mathbb{R}^{2}$ with smooth boundary and $a C^{\infty}$ function $a(x, y)$ such that:

1. The operator $A$ has n-dimensional kernel;

2. For every $m \geq 0$ there is an open neighborhood $\mathcal{B}$ of 0 in $J_{0}^{k}\left(X_{1}\right)$ such that every $C^{m}$-family of jets in $\mathcal{B}$ can be realized in (1) by a family of nonlinearities in $\mathcal{E}\left(q_{1}, \ldots, q_{k}\right)$. 
Choosing $q_{l}=l$ for all $l=1, \ldots, k$, we obtain [11, Theorem 2.4]. Similarly as in [11, Corollary 2.5], Theorem 2 implies that a dense set of vector fields in $\mathbb{R}^{n}$ can be realized, up to flow equivalence, in equation (1) by nonlinearities which have prescribed polynomial dependence on $u$ and $u_{y}$. More precisely, in the case of linear gradient dependence, we have the following result:

Theorem 3. Let $n \in \mathbb{N}$ be arbitrary, $B$ be an open ball in $\mathbb{R}^{n}$ containing 0 , and ${ }_{0} C^{1}(\bar{B})$ be the Banach space of all $C^{1}$-maps $g: \bar{B} \rightarrow \mathbb{R}^{n}$ with $g(0)=0$, endowed with the $C^{1}$-norm. There is a dense set $\mathcal{D}$ in ${ }_{0} C^{1}(\bar{B})$ with the property that for every $g \in \mathcal{D}$ there exist a smooth bounded domain $\Omega \subset \mathbb{R}^{2}$, a smooth function $a=a(x, y)$ and a nonlinearity $f$ of the form

$$
f(x, y, s, w)=\sum_{l=1}^{k} a_{l}(x, y) s^{l-1} w, \quad(x, y, s, w) \in \mathbb{R}^{4},
$$

for some $k \in \mathbb{N}$, such that the flow of the equation

$$
\dot{v}=g(v), \quad v \in B
$$

is $C^{1}$-equivalent to the flow of equation (1) (with the given $\Omega$, a and $f$ ) restricted to some locally invariant (center) manifold.

Proof. This theorem follows from Theorem 2 in exactly the same way as [11, Corollary 2.5] follows from [11, Theorem 2.4].

Remark. In the statements of Theorems 1 - 3 the coefficients $a_{l}(x, y)$ can be assumed $C^{\infty}$-smooth. This follows from the remark following condition (IC) below.

Readers interested in other realization results are referred to the References. In particular, the recent paper [2] proves realization of jets and dense vector fields in the class of (nonpolynomial) spatially homogeneous nonlinearities, i.e. functions of the form $f=f(u, \nabla u)$. Such functions do depend on $\nabla u$ in a complicated, largely arbitrary way, but, on the other hand, they are independent of the space variables. Note that neither the methods in [2] nor the methods used in the present paper seem to yield any general jet realization results in the class of functions which are spatially homogeneous and have prescribed polynomial dependence on $u$ and $\nabla u$.

Let us now proceed to the proof of Theorems 1 and 2 .

Identifying functions $f=f(x, y, s, w)=\sum_{l=1}^{k} a_{l}(x, y) s^{l-q_{l}} w^{q_{l}} \in \mathcal{E}\left(q_{1}, \ldots, q_{k}\right)$ with $\left(a_{l}\right)_{1 \leq l \leq k}$ (thus identifying $\mathcal{E}\left(q_{1}, \ldots, q_{k}\right)$ with the Hilbert space $\left.\left(H^{2}(\Omega)\right)^{k}\right)$ and using the arguments contained in sections 2 and 3 of [11], we see that, given a domain $\Omega \subset \mathbb{R}^{2}$ and a function $a$, statements (2) of Theorem 1 and Theorem 2 hold provided the following surjectivity condition is satisfied:

(SC). For every polynomial function $H: X_{1} \rightarrow X_{1}$ of degree $\leq k, H(0)=0$, there is an $f \in \mathcal{E}\left(q_{1}, \ldots, q_{k}\right)$ such that

$$
\sum_{j=1}^{n} \phi_{j} \int_{\Omega} \phi_{j}(x, y) f\left(x, y, u_{1}(x, y), u_{1 y}(x, y)\right) d x d y=H\left(u_{1}\right)
$$

for all $u_{1} \in X_{1}$.

We shall now find an equivalent, but more convenient form of this condition.

Let us introduce coordinates $\left(r_{1}, \ldots, r_{n}\right)$ in $X_{1}$, with respect to the basis $\phi_{1}, \ldots$, $\phi_{n}$, so that $u_{1} \in X_{1}$ can be written in the form $u_{1}=r_{1} \phi_{1}+\cdots+r_{n} \phi_{n}$. Then, 
if $H \in J_{0}^{k}\left(X_{1}\right)$, there are uniquely determined real coefficients $\rho_{j \beta}, j=1, \ldots, n$, $\beta \in \mathbb{N}^{n},|\beta| \leq k$, such that

$$
H\left(\sum_{i=1}^{n} r_{i} \phi_{i}\right)=\sum_{j=1}^{n}\left(\sum_{l=1}^{k} \sum_{|\beta|=l} \rho_{j \beta} r^{\beta}\right) \phi_{j}
$$

Thus (4) reads

$$
\int_{\Omega} \phi_{j}(x, y) f\left(x, y, \sum_{i=1}^{n} r_{i} \phi_{i}(x, y), \sum_{i=1}^{n} r_{i} \phi_{i y}(x, y)\right) d x d y=\sum_{l=1}^{k} \sum_{|\beta|=l} \rho_{j \beta} r^{\beta},
$$

for $j=1, \ldots, n$. The nonlinearity $f$ has the form

$$
f\left(x, y, u, u_{y}\right)=\sum_{l=1}^{k} a_{l}(x, y) u^{l-q_{l}} u_{y}^{q_{l}},
$$

so (4) becomes

$$
\begin{aligned}
\int_{\Omega} \phi_{j} \sum_{l=1}^{k} a_{l}\left(\sum_{j=1}^{n} r_{j} \phi_{j}\right)^{l-q_{l}}\left(\sum_{i=1}^{n} r_{i} \phi_{i y}\right)^{q_{l}} d x d y=\sum_{\substack{l=1 \\
j}}^{k} \sum_{|\beta|=l} \rho_{j \beta} r^{\beta}, \ldots, n .
\end{aligned}
$$

Consequently (SC) is satisfied, provided we can find functions $a_{1}, \ldots, a_{k} \in H^{2}(\Omega)$ such that (5) holds for all $\left(r_{1}, \ldots, r_{n}\right) \in \mathbb{R}^{n}$. The left hand side of (5) can be manipulated in the following way:

$$
\begin{aligned}
& \int_{\Omega} \phi_{j} \sum_{l=1}^{k} a_{l}\left(\sum_{j=1}^{n} r_{j} \phi_{j}\right)^{l-q_{l}}\left(\sum_{i=1}^{n} r_{i} \phi_{i y}\right)^{q_{l}} d x d y \\
& =\int_{\Omega} \phi_{j} \sum_{l=1}^{k} a_{l}\left(\sum_{|\alpha|=l-q_{l}} \frac{\left(l-q_{l}\right) !}{\alpha !} r^{\alpha} \phi^{\alpha}\right)\left(\sum_{|\gamma|=q_{l}} \frac{q_{l} !}{\gamma !} r^{\gamma} \phi_{y}^{\gamma}\right) d x d y \\
& =\sum_{l=1}^{k} \sum_{|\alpha|=l-q_{l}} \sum_{|\gamma|=q_{l}} \frac{\left(l-q_{l}\right) !}{\alpha !} \frac{q_{l} !}{\gamma !} r^{\alpha+\gamma} \int_{\Omega} a_{l} \phi_{j} \phi^{\alpha} \phi_{y}^{\gamma} d x d y \\
& =\sum_{l=1}^{k} \sum_{|\beta|=l}\left(\sum_{\substack{\alpha+\gamma=\beta \\
|\alpha|=l-q_{l},|\gamma|=q_{l}}} \frac{\left(l-q_{l}\right) ! q_{l} !}{\alpha ! \gamma !} \int_{\Omega} a_{l} \phi_{j} \phi^{\alpha} \phi_{y}^{\gamma} d x d y\right) r^{\beta} .
\end{aligned}
$$

Equating coefficients, we therefore see that (SC) is satisfied if and only if for every $l=1, \ldots, k$, for all $\rho_{j \beta} \in \mathbb{R}, j=1, \ldots, n, \beta \in \mathbb{N}_{0}^{n},|\beta|=l$, there is $a_{l}(x, y) \in H^{2}(\Omega)$ such that, for all $j=1, \ldots, n$, and for all $\beta \in \mathbb{N}_{0}^{n}$ with $|\beta|=l$,

$$
\int_{\Omega} a_{l}\left(\sum_{\substack{\alpha+\gamma=\beta \\|\alpha|=l-q_{l},|\gamma|=q_{l}}} \frac{\left(l-q_{l}\right) ! q_{l} !}{\alpha ! \gamma !} \phi_{j} \phi^{\alpha} \phi_{y}^{\gamma}\right) d x d y=\rho_{j \beta} .
$$


Using the density of $H^{2}(\Omega)$ in $L^{2}(\Omega)$, it is easy to see that this latter condition is satisfied if and only if for for every $l=1, \ldots, k$ the functions

$$
\left\{\sum_{\substack{\alpha+\gamma=\beta \\|\alpha|=l-q_{l},|\gamma|=q_{l}}} \frac{\left(l-q_{l}\right) ! q_{l} !}{\alpha ! \gamma !} \phi_{j} \phi^{\alpha} \phi_{y}^{\gamma}\right\}_{\substack{j=1, \ldots, n \\|\beta|=l}}
$$

are linearly independent.

Now we introduce the following notations: given $\gamma, \beta \in \mathbb{N}_{0}^{n}$, we say that $\gamma \leq \beta$ iff $\gamma_{i} \leq \beta_{i}, i=1, \ldots, n$. Moreover, set

$$
\epsilon_{j}:=(\underbrace{0, \ldots, 0,1}_{j}, 0, \ldots, 0) \in \mathbb{N}_{0}^{n} .
$$

With these notations, the independence condition reads:

(IC). For every $l=1, \ldots, k$ and for every $q, 1 \leq q \leq l$, the functions

$$
\left\{\sum_{\substack{\gamma \leq \beta \\|\gamma|=q}} \frac{1}{\gamma !(\beta-\gamma) !} \phi^{\beta-\gamma+\epsilon_{j}} \phi_{y}^{\gamma}\right\}_{\substack{j=1, \ldots, n \\|\beta|=l}}
$$

are linearly independent.

Remark. If (IC) is satisfied then the functions $a_{l}$ in (6) can actually be chosen to belong to any dense subspace of $L^{2}(\Omega)$, e.g. they can be chosen as smooth as $\Omega$ is. On the other hand, these functions cannot be chosen to be constant, in general. The reason for this is that the subspace of functions in $\mathcal{E}\left(q_{1}, \ldots, q_{k}\right)$ with spatially constant coefficients has dimension $k$, while, for $\operatorname{dim} X_{1}>1$, the space of jets $J_{0}^{k}\left(X_{1}\right)$ has dimension $>k$, so the surjectivity condition (SC) is not satisfied in this case.

It follows that in order to prove Theorems 1 and 2 we only have to find a smooth convex bounded domain $\Omega$ and a smooth function $a(x, y)$ in such a way that condition (IC) is satisfied.

As in [11] we begin by considering the eigenvalue problem

$$
\begin{aligned}
& u_{x x}+u_{y y}+a(x) u+\mu u=0 \quad \text { in } \Omega, \\
& u=0 \text { in } \partial \Omega,
\end{aligned}
$$

on the square $\Omega=] 0, \pi[\times] 0, \pi\left[\right.$. Let $\lambda_{j}(a), j \in \mathbb{N}$, be the increasing sequence of the eigenvalues of the problem

$$
\begin{aligned}
u_{x x}+a(x) u+\lambda u=0 & \text { in }] 0, \pi[, \\
u=0 & \text { in }\{0, \pi\},
\end{aligned}
$$

and $\psi_{j}(a), j \in \mathbb{N}$, be the corresponding $L^{2}(0, \pi)$-normalized eigenfunctions. Choose positive integers $m_{1}>m_{2} \cdots>m_{n}$ which are $(k+1)$-conditionally rationally independent. As in [11], let $a=a(x)$ be a $C^{\infty}$ function on $[0, \pi]$ such that

$$
\begin{gathered}
a(x)=0 \quad \text { on }[0, \delta], \text { for some } \delta>0 ; \\
\lambda_{j}(a)=-m_{j}^{2} \quad \text { for } j=1, \ldots, n ; \\
\lambda_{n+1}(a)>0 .
\end{gathered}
$$


It follows that the eigenvalue problem (7) has $\mu=0$ as an eigenvalue. The corresponding eigenspace is $n$-dimensional and is spanned by the eigenfunctions

$$
\phi_{j}(x, y)=\sin \left(m_{j} y\right) \psi_{j}(a)(x), \quad j=1, \ldots, s,(x, y) \in \Omega .
$$

We have the following

Proposition 1. The eigenfunctions in (8) satisfy the independence condition (IC).

Proof. Fix $l=1, \ldots, k$ and suppose

$$
\sum_{\substack{j=1, \ldots, n \\|\beta|=l}} C_{j \beta} \sum_{\substack{\gamma \leq \beta \\|\gamma|=q}} \frac{1}{\gamma !(\beta-\gamma) !} \phi^{\beta-\gamma+\epsilon_{j}} \phi_{y}^{\gamma}=0 .
$$

Since $a(x)=0$ for $x \in[0, \delta]$, we see that

$$
\phi_{j}(x, y)=\eta_{j}(x) \xi_{j}(y), \quad x \in[0, \delta], y \in[0, \pi],
$$

where

$$
\begin{gathered}
\eta_{j}(x)=d_{j} \sinh \left(m_{j} x\right), \quad d_{j} \neq 0, \\
\xi_{j}(y)=\sin \left(m_{j} y\right)
\end{gathered}
$$

for $j=1, \ldots, n$. Set

$$
\begin{gathered}
\eta(x)=\left(\eta_{1}(x), \ldots, \eta_{n}(x)\right), \\
\xi(y)=\left(\xi_{1}(y), \ldots, \xi_{n}(y)\right) .
\end{gathered}
$$

Thus, by analyticity,

$$
\begin{gathered}
\sum_{\substack{c \in \mathbb{N}^{n} \\
|c|=l+1}} \sum_{\substack{j=1, \ldots, n \\
\beta \mid=l \\
\beta+\epsilon_{j}=c}} C_{j \beta} \sum_{\substack{\gamma \leq \beta \\
|\gamma|=q}} \frac{1}{\gamma !(\beta-\gamma) !} \phi^{\beta-\gamma+\epsilon_{j}}(x, y) \phi_{y}^{\gamma}(x, y) \\
=\sum_{\substack{c \in \mathbb{N}^{n} \\
|c|=l+1}} \eta^{c}(x) \sum_{\substack{j=1, \ldots, n \\
|\beta|=l \\
\beta+\epsilon_{j}=c}} C_{j \beta} \sum_{\substack{\gamma \leq \beta \\
|\gamma|=q}} \frac{1}{\gamma !(\beta-\gamma) !} \xi^{c-\gamma}(y) \xi_{y}^{\gamma}(y)=0 \quad \text { for }(x, y) \in \mathbb{R}^{2} .
\end{gathered}
$$

By the arguments in [11, pp. 41-42] we obtain that the functions $\left(\eta^{c}\right)_{|c|=l+1}$ are linearly independent, so

$$
\begin{gathered}
\sum_{\substack{j=1, \ldots, n \\
|\beta|=l \\
\beta+\epsilon_{j}=c}} C_{j \beta} \sum_{\substack{\gamma \leq \beta \\
|\gamma|=q}} \frac{1}{\gamma !(\beta-\gamma) !} \xi^{c-\gamma}(y) \xi_{y}^{\gamma}(y) \\
=\sum_{|\gamma|=q}\left(\sum_{\substack{j=1, \ldots, n \\
|\beta|=l, \beta \geq \gamma \\
\beta+\epsilon_{j}=c}} C_{j \beta} \frac{1}{\gamma !(\beta-\gamma) !}\right) \xi^{c-\gamma}(y) \xi_{y}^{\gamma}(y)=0 \quad \text { for }|c|=l+1 \text { and } y \in \mathbb{R} .
\end{gathered}
$$

By Lemma 1 in the Appendix the functions $\left(\xi^{c-\gamma} \xi_{y}^{\gamma}\right)_{|\gamma|=q}$ are linearly independent, so

$$
\sum_{\substack{j=1, \ldots, n \\|\beta|=l, \beta \geq \gamma \\ \beta+\epsilon_{j}=c}} C_{j \beta} \frac{1}{(\beta-\gamma) !}=0 \quad \text { for }|\gamma|=q \text { and }|c|=l+1 .
$$


Now it is easy to see that

so

$$
\begin{aligned}
\sum_{\substack{j=1, \ldots, n \\
|\beta|=l, \beta \geq \gamma \\
\beta+\epsilon_{j}=c}} C_{j \beta} \frac{1}{(\beta-\gamma) !} & =\sum_{\substack{j=1, \ldots, n \\
\gamma+\epsilon_{j} \leq c}} C_{j, c-\epsilon_{j}} \frac{1}{\left(c-\epsilon_{j}-\gamma\right) !} \\
& =\sum_{\substack{j=1, \ldots, n \\
\gamma+\epsilon_{j} \leq c}} C_{j, c-\epsilon_{j}} \frac{\left(c_{j}-\gamma_{j}\right)}{(c-\gamma) !},
\end{aligned}
$$

$$
\sum_{\substack{j=1, \ldots, n \\ \gamma+\epsilon_{j} \leq c}} C_{j, c-\epsilon_{j}}\left(c_{j}-\gamma_{j}\right)=0 \text { for }|\gamma|=q \text { and }|c|=l+1 .
$$

Now fix $c \in \mathbb{N}_{0}^{n},|c|=l+1$. If $\gamma \not \leq c$, then the sum in (9) is over an empty set of elements; if $\gamma \leq c$, but $\gamma+\epsilon_{j} \not \leq c$, then $\gamma_{j}+1 \not \leq c_{j}$ and so $\gamma_{j}=c_{j}$. Thus (9) is equivalent to the statement that for every $c \in \mathbb{N}_{0}^{n}$ with $|c|=\sum_{i=1}^{n} c_{i}=l+1$

$$
\sum_{\substack{j=1, \ldots, n \\ \epsilon_{j} \leq c}} C_{j, c-\epsilon_{j}}\left(c_{j}-\gamma_{j}\right)=0 \quad \text { whenever } \gamma \leq c \text { and }|\gamma|=q
$$

Let $c \in \mathbb{N}_{0}^{n}$ with $|c|=l+1$ be arbitrary. We will show that (10) implies that $C_{j, c-\epsilon_{j}}=0$ for all $j$ such that $c \geq \epsilon_{j}$. This will conclude the proof of the proposition. Permuting components, we may assume that, for some $s, 1 \leq s \leq n, c_{j} \geq 1$ if $1 \leq j \leq s$ and $c_{j}=0$ if $s+1 \leq j \leq n$. Then whenever $\gamma \leq c$, we also have $\gamma_{j}=0$ for $s+1 \leq j \leq n$. Therefore we only have to prove the following assertion:

(A). If $s \in \mathbb{N}, q \in \mathbb{N}, c \in \mathbb{N}^{s}$ and $a \in \mathbb{R}^{s}$ are such that $q<|c|$ and

$$
\sum_{j=1}^{s} a_{j}\left(c_{j}-\gamma_{j}\right)=0 \quad \text { whenever } \gamma \in \mathbb{N}_{0}^{s}, \gamma \leq c \text { and }|\gamma|=q,
$$

then $a_{j}=0$ for all $j=1, \ldots, s$.

In order to prove (A) we apply Lemma 2 in the Appendix with $p=|c|-q$. Let $x^{i}, i=1, \ldots, s$, be as in that lemma, and set $\gamma^{i}:=c-x^{i}, i=1, \ldots, s$. It follows that $\left|\gamma^{i}\right|=p$ and $\gamma^{i} \leq c$ for all $i$ and that the matrix $\left(c_{j}-\gamma_{j}^{i}\right)_{1 \leq i, j \leq s}=\left(x_{j}^{i}\right)_{1 \leq i, j \leq s}$ is regular. Assertion (A) follows immediately, and so the proposition is proved.

So far we have proved that there is a smooth function $a=a(x)$ (i.e. independent of $y$ ) such that the surjectivity condition (SC) is satisfied. In particular, taking $q_{l}=l$ for all $l=1, \ldots, k$, we obtain the results in $[11$, section IV A] as a special case. However, we also claim that there are a smooth convex bounded domain $\Omega$ and a smooth function $a(x, y)$ such that condition (SR) is satisfied. In order to prove this claim, we proceed exactly as in [11, section IV B] but defining the functions $\chi_{j \beta}$ as follows:

$$
\chi_{j \beta}=\sum_{\substack{\gamma \leq \beta \\|\gamma|=q}} \frac{1}{\gamma !(\beta-\gamma) !} \phi^{\beta-\gamma+\epsilon_{j}} \phi_{y}^{\gamma} \quad \text { for } j=1, \ldots, n,|\beta|=l
$$

with $\phi_{j}=\phi_{j}(a, s, x, y)$, where $a$ and $s$ are as in [11, section IV B]. No other changes are necessary. This proves the claim and completes the proof of Theorems 1 and 2 . 


\section{APPENDIX}

We shall now prove the auxiliary results used in the proof of Proposition 1.

Recall that $m_{1}, \ldots, m_{n} \in \mathbb{R}$ are said to be $M$-conditionally rationally independent iff whenever $\gamma_{j} \in \mathbb{Z}, j=1, \ldots, n$, are such that $\left|\gamma_{j}\right| \leq M$, for $j=1, \ldots, n$ and

$$
\sum_{j=1}^{n} \gamma_{j} m_{j}=0
$$

then $\gamma_{j}=0$, for $j=1, \ldots, n$.

Lemma 1. Suppose that $d \in \mathbb{N}$ and $m_{1}, \ldots, m_{n} \in \mathbb{R}$ are $d$-conditionally rationally independent. For every multi-index $\gamma \in \mathbb{N}_{0}^{n}$, define the function $f_{\gamma}$ by

$$
f(x)=\prod_{k=1}^{n} \cot \left(m_{k} x\right)^{\gamma_{k}} .
$$

Then the functions $\left\{f_{\gamma}\right\}_{|\gamma| \leq d}$ are linearly independent on every open interval $I \subset \mathbb{R}$ on which these functions are defined.

Proof. We have to prove that whenever

$$
\sum_{|\gamma| \leq d} a_{\gamma} \mathrm{i}^{-|\gamma|} f_{\gamma}=0 \quad \text { on } I
$$

then

$$
a_{\gamma}=0 \text { for all }|\gamma| \leq d
$$

Since

$$
\cot y=\mathrm{i} \frac{1+e^{-2 \mathrm{i} y}}{1-e^{-2 \mathrm{i} y}}
$$

we obtain that (11) is equivalent to

$$
\sum_{|\gamma| \leq d} a_{\gamma} \prod_{k=1}^{n} \frac{\left(1+e^{-2 \mathrm{i} m_{k} x}\right)^{\gamma_{k}}}{\left(1-e^{-2 \mathrm{i} m_{k} x}\right)^{\gamma_{k}}}=0 \quad \text { for } x \in I .
$$

By analyticity, (13) is equivalent to

$$
\sum_{|\gamma| \leq d} a_{\gamma} \prod_{k=1}^{n}\left(1+e^{m_{k} x}\right)^{\gamma_{k}} \prod_{k=1}^{n}\left(1-e^{m_{k} x}\right)^{d-\gamma_{k}}=0 \quad \text { for } x \in \mathbb{C} .
$$

Using the notation

$$
\left(\begin{array}{l}
\gamma \\
\mathbf{k}
\end{array}\right)=\prod_{j=1}^{n}\left(\begin{array}{l}
\gamma_{j} \\
k_{j}
\end{array}\right)
$$

for $\mathbf{k}=\left(k_{1}, \ldots, k_{n}\right) \in \mathbb{N}_{0}^{n}$ and setting

$$
\mathbf{d}=(\underbrace{d, \ldots, d}_{n \text { times }}),
$$

we see that the right hand side of (14) is equal to

$$
\sum_{|\gamma| \leq d} a_{\gamma} \sum_{\substack{\mathbf{k}, \mathbf{l} \in \mathbb{N}_{0}^{n} \\
\mathbf{k} \leq \gamma, \mathbf{l} \leq \mathbf{d}-\gamma}}(-1)^{|\mathbf{l}|}\left(\begin{array}{l}
\gamma \\
\mathbf{k}
\end{array}\right)\left(\begin{array}{c}
\mathbf{d}-\gamma \\
\mathbf{l}
\end{array}\right) e^{\sum_{j} m_{j}\left(k_{j}+l_{j}\right) x} .
$$


Thus (14) is equivalent to

$$
\sum_{\mathbf{r} \in \mathbb{N}_{0}^{n}}\left(\sum_{|\gamma| \leq d} a_{\gamma} \sum_{\substack{\mathbf{k}, \mathbf{l} \in \mathbb{N}^{n} \\
\mathbf{k} \leq \gamma, \mathbf{l} \leq \mathbf{d}-\gamma \\
\mathbf{k}+\mathbf{l}=\mathbf{r}}}(-1)^{|\mathbf{l}|}\left(\begin{array}{c}
\gamma \\
\mathbf{k}
\end{array}\right)\left(\begin{array}{c}
\mathbf{d}-\gamma \\
\mathbf{1}
\end{array}\right)\right) e^{\left(\sum_{j} m_{j} r_{j}\right) x}=0 \quad \text { on } \mathbb{C} .
$$

Since $m_{1}, \ldots, m_{n}$ are $d$-conditionally rationally independent, it follows that

$$
\sum_{j} m_{j} r_{j} \neq \sum_{j} m_{j} s_{j} \quad \text { whenever } \mathbf{r} \neq \mathbf{s},\left|r_{j}\right| \leq d,\left|s_{j}\right| \leq d \text { for } j=1, \ldots, n .
$$

The linear independence of the functions $\left(e^{c x}\right)_{c \in \mathbb{R}}$ now implies that (11) is equivalent to

$$
\sum_{|\gamma| \leq d} a_{\gamma} \sum_{\substack{\mathbf{k}, \mathbf{l} \in \mathbb{N}_{0}^{n} \\
\mathbf{k} \leq \gamma, \mathbf{1} \mathbf{d}-\gamma \\
\mathbf{k}+\mathbf{l}=\mathbf{r}}}(-1)^{|\mathbf{1}|}\left(\begin{array}{l}
\gamma \\
\mathbf{k}
\end{array}\right)\left(\begin{array}{c}
\mathbf{d}-\gamma \\
\mathbf{l}
\end{array}\right)=0 \quad \text { whenever }\left|r_{j}\right| \leq d \text { for } j=1, \ldots, n .
$$

On the other hand, using similar arguments, we also obtain the following equivalences:

if and only if

$$
a_{\gamma}=0 \quad \text { for all }|\gamma| \leq d
$$

$$
\sum_{|\gamma| \leq d} a_{\gamma} y^{\gamma}=0 \quad \text { for } y \in \mathbb{R}^{n}
$$

if and only if

$$
\sum_{|\gamma| \leq d} a_{\gamma} \prod_{k=1}^{n} \frac{\left(1+x_{k}\right)^{\gamma_{k}}}{\left(1-x_{k}\right)^{\gamma_{k}}}=0 \quad \text { whenever } x \in \mathbb{R}^{n} \text { with } x_{j} \neq 1, \text { for } j=1, \ldots n
$$

if and only if

$$
\sum_{|\gamma| \leq d} a_{\gamma} \prod_{k=1}^{n}\left(1+x_{k}\right)^{\gamma_{k}} \prod_{k=1}^{n}\left(1-x_{k}\right)^{d-\gamma_{k}}=0 \quad \text { for } x \in \mathbb{R}^{n}
$$

if and only if

$$
\sum_{\mathbf{r} \in \mathbb{N}_{0}^{n}}\left(\sum_{|\gamma| \leq d} a_{\gamma} \sum_{\substack{\mathbf{k}, \mathbf{l} \in \mathbb{N}_{0}^{n} \\
\mathbf{k} \leq \gamma, \mathbf{l} \leq \mathbf{d}-\gamma \\
\mathbf{k}+\mathbf{l}=\mathbf{r}}}(-1)^{|\mathbf{l}|}\left(\begin{array}{l}
\gamma \\
\mathbf{k}
\end{array}\right)\left(\begin{array}{c}
\mathbf{d}-\gamma \\
\mathbf{l}
\end{array}\right)\right) x^{\mathbf{r}}=0 \quad \text { for } x \in \mathbb{R}^{n}
$$

if and only if

$$
\sum_{|\gamma| \leq d} a_{\gamma} \sum_{\substack{\mathbf{k}, \mathbf{l} \in \mathbb{N}_{0}^{n} \\
\mathbf{k} \leq \gamma, \mathbf{l} \leq \mathbf{d}-\gamma \\
\mathbf{k}+\mathbf{l}=\mathbf{r}}}(-1)^{|\mathbf{1}|}\left(\begin{array}{l}
\gamma \\
\mathbf{k}
\end{array}\right)\left(\begin{array}{c}
\mathbf{d}-\gamma \\
\mathbf{l}
\end{array}\right)=0 \quad \text { whenever }\left|r_{j}\right| \leq d \text { for } j=1, \ldots, n .
$$

We thus obtain that (11) is equivalent to (16) and (16) is equivalent to (12). This concludes the proof of the lemma. 
Remark. The lemma may not hold if $m_{1}, \ldots, m_{n} \in \mathbb{R}$ are not $d$-conditionally rationally independent. In fact, using the formula

$$
2 \cot 2 x \cot x-(\cot x)^{2}+1=0
$$

and choosing $n=2, m_{1}=2, m_{2}=1$ and $d=2$, we see that $\left\{f_{\gamma}\right\}|\gamma| \leq d$ are linearly dependent on every open interval $I \subset \mathbb{R}$ on which these functions are defined.

Lemma 2. If $s \in \mathbb{N}, p \in \mathbb{N}$ and $c \in \mathbb{N}^{s}$ are such that $p<|c|$, then there exist vectors $x^{1}, \ldots, x^{s} \in \mathbb{N}_{0}^{s}$ such that:

1. $x^{1}, \ldots, x^{s}$ are linearly independent;

2. $x^{i} \leq c, i=1, \ldots, s$;

3. $\left|x^{i}\right|=p, i=1, \ldots, s$.

Proof. Define

$$
C:=\left\{x \in \mathbb{R}^{s}|| x \mid:=\sum_{j=1}^{s} x_{j}=p, \text { and } 0 \leq x \leq c\right\} .
$$

$C$ is convex, closed and bounded, so $C$ is the convex hull of the set $C^{*}$ of the extremal points of $C$.

We claim that for every $x \in C^{*}$ there is an index $i=i(x)$ such that $x_{j} \in\left\{0, c_{j}\right\}$ for all $j$ with $j \neq i$. In fact, suppose there are $x \in C^{*}$ and indices $i$ and $j$ with $i \neq j, 0<x_{i}<c_{i}$ and $0<x_{j}<c_{j}$. Define $y, z \in \mathbb{R}^{s}$ in the following way:

$$
\begin{gathered}
y_{k}=z_{k}=x_{k}, \quad k \neq i, j, \\
y_{i}=x_{i}+\epsilon, \quad y_{j}=x_{j}-\epsilon, \\
z_{i}=x_{i}-\epsilon, \quad z_{j}=x_{j}+\epsilon .
\end{gathered}
$$

We have $|y|=|z|=p=|x|$, and, if $\epsilon$ is sufficiently small, $y, z \in C$; but $x=$ $(1 / 2)(y+z)$, contradicting the fact that $x \in C^{*}$. This proves the claim. Since $c \in \mathbb{N}_{0}^{s}$, the claim implies for every $x \in C^{*}$ that $x_{j} \in \mathbb{N}_{0}$ for all $j \neq i$, where $i=i(x)$. Since $|x|=p \in \mathbb{N}$, it also follows that $x_{i} \in \mathbb{N}_{0}$. So we conclude that, if $x \in C^{*}$, then $x \in \mathbb{N}_{0}^{s}$. If we show that $\operatorname{dim} \operatorname{span} C^{*}=s$, then the lemma will be proved. Consider the hyperplane

$$
H:=\left\{x \in \mathbb{R}^{s}|| x \mid=\sum_{j=1}^{s} x_{i}=0\right\} .
$$

Clearly $\operatorname{dim} H=s-1$. Let $\lambda:=p /|c|$ (notice that $0<\lambda<1$ ), and set $a:=\lambda c$; then $|a|=p$, and, since $0<a_{i}<c_{i}$ for all $i$, it follows that $a \leq c$, so that $a \in C$. Now write $C^{*}$ as $C^{*}=\left\{x_{\alpha}\right\}_{\alpha \in A}$. Then there are $\left\{\lambda_{\alpha}\right\}_{\alpha \in A}, \lambda_{\alpha} \geq 0$ for all $\alpha, \lambda_{\alpha}=0$ for almost all $\alpha, \sum_{\alpha} \lambda_{\alpha}=1$, such that $a=\sum_{\alpha} \lambda_{\alpha} x_{\alpha}$. Let $y^{1}, \ldots, y^{s-1} \in H$ be linearly independent. Choose a sufficiently small $\delta>0$, so that $0<\delta y_{i}^{j}+a_{i}<c_{i}$, for all $j=1, \ldots, s-1, i=1, \ldots, s$. Then $\delta y^{j}+a \in C$ for $j=1, \ldots, s-1$. For every $j=1, \ldots, s-1$ there are $\left\{\lambda_{\alpha}^{j}\right\}_{\alpha \in A}$ such that $\lambda_{\alpha}^{j} \geq 0$ for all $\alpha, \lambda_{\alpha}^{j}=0$ for almost all $\alpha, \sum_{\alpha} \lambda_{\alpha}^{j}=1$ and $y^{j}=\sum_{\alpha} \lambda_{\alpha}^{j} x_{\alpha}$. It follows that

$$
y^{j}=\sum_{\alpha} \frac{\left(\lambda_{\alpha}^{j}-\lambda_{\alpha}\right)}{\delta} x_{\alpha}
$$


so that $y^{j} \in \operatorname{span} C^{*}$ for $j=1, \ldots, s-1$. If $a \in \operatorname{span}\left\{y_{1}, \ldots, y_{s-1}\right\}$, then $a \in H$, and then $|a|=0$, but this is impossible since $|a|=p>0$. Thus we obtain

$$
\operatorname{span} C^{*}=\operatorname{span}\left\{a, y_{1}, \ldots, y_{s-1}\right\}=\mathbb{R}^{s} .
$$

This concludes the proof.

\section{REFERENCES}

1. E. N. Dancer, On the existence of two-dimensional invariant tori for scalar parabolic equations with time periodic coefficients, Annali Scuola Norm. Sup. Pisa 43 (1991), 455 - 471. MR 93c: 35077

2. E. N. Dancer and P. Poláčik, Realization of vector fields and dynamics of spatially homogeneous parabolic equations, preprint.

3. T. Faria and L. Magalhães, Realisation of ordinary differential equations by retarded functional differential equations in neighborhoods of equilibrium points, Proc. Roy. Soc. Edinburgh Sect. A 125 (1995), 759-776. MR 97g:34088

4. B. Fiedler and P. Poláčik, Complicated dynamics of scalar reaction-diffusion equations with a nonlocal term, Proc. Roy. Soc. Edinburgh Sect. A 115 (1990), 167-192. MR 91g:45008

5. B. Fiedler and B. Sandstede, Dynamics of periodically forced parabolic equations on the circle, Ergodic Theory Dynamical Systems 12 (1992), 559-571. MR 93h:35103

6. J. K. Hale, Flows on centre manifolds for scalar functional differential equations, Proc. Roy. Soc. Edinburgh Sect. A 101 (1985), 193-201. MR 87d:34117

7. P. Poláčik, Complicated dynamics in scalar semilinear parabolic equations in higher space dimension, J. Differential Equations 89 (1991), 244-271. MR 92c:35063

8. P. Poláčik, Imbedding of any vector field in a scalar semilinear parabolic equation, Proc. Amer. Math. Soc. 115 (1992), 1001-1008. MR 92j:35099

9. P. Poláčik, Realization of any finite jet in a scalar semilinear equation on the ball in $\mathbb{R}^{3}$, Ann. Scuola Norm. Sup. Pisa XVII (1991), 83-102. MR 92g:35107

10. P. Poláčik, Realization of the dynamics of ODEs in scalar parabolic PDEs, Tatra Mountains Math. Publ. 4 (1994), 179-185. MR 95f:35117

11. P. Poláčik, High-dimensional $\omega$-limit sets and chaos in scalar parabolic equations, J. Differential Equations 119 (1995), 24-53. MR 96h:35092

12. P. Poláčik, Reaction-diffusion equations and realization of gradient vector fields, Proc. Equadiff '95 (to appear).

13. P. Poláčik and K. P. Rybakowski, Imbedding vector fields in scalar parabolic Dirichlet BVPs, Ann. Scuola Norm. Sup. Pisa XXI (1995), 737-749. MR 97a:35124

14. P. Poláčik and K. P. Rybakowski, Nonconvergent bounded trajectories in semilinear heat equations, J. Differential Equations 124 (1995), 472- 494. MR 96m:35176

15. M. Prizzi and K. P. Rybakowski, Inverse problems and chaotic dynamics of parabolic equations on arbitrary spatial domains, J. Differential Equations 142 (1998), 17-53.

16. K. P. Rybakowski, Realization of arbitrary vector fields on center manifolds of parabolic Dirichlet BVPs, J. Differential Equations 114 (1994), 199-221. MR 95j:35114

17. K. P. Rybakowski, Realization of arbitrary vector fields on invariant manifolds of delay equations, J. Differential Equations 114 (1994), 222-231. MR 96e:34121

18. K. P. Rybakowski, The center manifold technique and complex dynamics of parabolic equations, Topological Methods in Differential Equations and Inclusions (A. Granas, M. Frigon, eds.), NATO ASI Series, vol. 472, Kluwer Academic Publishers, Dordrecht/Boston/London, 1995, pp. 411-446. MR 97b:35095

SiSSA, Via Beirut 2-4, 34013 Trieste, Italy

E-mail address: prizzi@sissa.it

Universität Rostock, Fachbereich Mathematik, Universitätsplatz 1, 18055 Rostock, Germany

E-mail address: krzysztof.rybakowski@mathematik.uni-rostock.de 